\title{
Efficacy of Multilayered Hepatocyte Sheet Transplantation for Radiation-Induced Liver Damage and Partial Hepatectomy in a Rat Model
}

\author{
Zhassulan Baimakhanov, Kosho Yamanouchi, Yusuke Sakai, Makiko Koike, Akihiko Soyama, Masaaki Hidaka, \\ Mitsuhisa Takatsuki, Fumihiko Fujita, Kengo Kanetaka, Tamotsu Kuroki, and Susumu Eguchi
}

Department of Surgery, Nagasaki University Graduate School of Biomedical Sciences, Sakamoto, Nagasaki, Japan

\begin{abstract}
Although cell sheet technology has recently been developed for use in both animal experiments and in the clinical setting, it remains unclear whether transplanted hepatocyte sheets improve the liver function in vivo. Radiation-induced liver damage (RILD) combined with partial hepatectomy (PH) has been reported to suppress the proliferation of host hepatocytes and induce critical liver failure. The aim of this study was to improve the liver function in the above-mentioned diseased rat model (RILD+PH) using multilayered hepatocyte sheet transplantation. In this study, we used Fischer rats as a donor for primary hepatocytes and dermal fibroblast isolation. Cocultured multilayered hepatocyte sheets were generated by disseminating hepatocytes onto fibroblasts cultured beforehand on temperature-responsive culture dishes. Four cell sheets were transplanted into the recipient rats subcutaneously. Prior to transplantation, RILD (50 Gy) with 2/3PH was induced in the recipients. The same model was applied in the control group without transplantation. The serum was collected each week. The rats in both groups were sacrificed at 2 months after transplantation for the histological analysis. Consequently, the serum albumin concentrations were significantly higher in the transplant group than in the control group $(54.3 \pm 9.6$ vs. $32.7 \pm 5.7 \mathrm{mg} / \mathrm{ml} ; p<0.01)$ after 2 months and comparable to the serum albumin levels in the normal rats $(58.1 \pm 6.4 \mathrm{mg} / \mathrm{ml})$. In addition, treatment with the transplanted sheets significantly improved the survival rate $(57 \%$ vs. $22 \%, p<0.05)$, and the hepatocyte sheets showed the storage of albumin, glycogen, and bile canaliculus structures. Some hepatocytes and fibroblasts were positive for Ki-67, and vascularization was observed around the cell sheets. Transplanted multilayered hepatocyte sheets can survive with additional proliferative activity, thereby maintaining the liver function in vivo for at least 2 months, providing metabolic support for rats with RILD.
\end{abstract}

Key words: Hepatocytes; Cell sheet; Transplantation; Rat diseased model; Metabolic support

\section{INTRODUCTION}

Liver transplantation is the only established treatment for patients with end-stage liver failure. However, due to the shortage of donors, alternative treatments are required. Hepatocyte transplantation (HT) and hepatic tissue engineering have been established as promising alternatives to liver transplantation. Many previously reported studies have shown that HT can be an alternative treatment to liver transplantation; however there remain unresolved issues before this type of treatment can be widely introduced in clinical practice $(1,6-8,13,17,19)$. Severe complications can occur after the intrasplenic or intraportal transplantation of hepatocytes, such as portal vein thrombosis and portal hypertension resulting from the obstruction of sinusoids and hepatic infarction $(8,13)$. Owing to the low survival rate of primary hepatocytes, it is necessary to transplant these cells as soon as possible after isolation $(6,28)$. In addition, it is difficult to obtain histological evidence of transplanted hepatocytes, which is required to detect possible cellular rejection (13).

Although cell sheet technology was recently developed and has already been applied in both in vitro and in vivo studies $(16,20-23)$, it is still unclear how transplanted hepatocyte sheets may improve the liver function and survival in diseased models. We previously reported our rapid fabricating technique for generating multilayered human hepatic cell sheets by using fibroblasts as feeder cells on temperature-responsive culture dishes (TRCDs) $(22,23)$. This technique shows the significant advantage of using cocultured hepatic cell sheets, with a more favorable survival and higher liver-specific function in vitro (22) and in vivo (23), compared with sheets consisting of only hepatocytes.

Received May 15, 2015; final acceptance September 15, 2015. Online prepub date: July 28, 2015.

Address correspondence to Susumu Eguchi, M.D., Ph.D., Department of Surgery, Nagasaki University Graduate School of Biomedical Sciences, 1-7-1 Sakamoto, Nagasaki 852-8501, Japan. Tel: +81-95-819-7316; Fax: +81-95-819-7319; E-mail: sueguchi@ nagasaki-u.ac.jp 
Radiation-induced liver damage (RILD) combined with partial hepatectomy $(\mathrm{PH})$ has been used in previous experiments with HT in animal models and shown to suppress the proliferation of host hepatocytes. In addition, transplanted hepatocytes are allowed to proliferate following $\mathrm{PH}$, which improves the survival rate $(7,17)$.

Considering previously reported results, in the current study we fabricated multilayered hepatocyte sheets from primary isolated rat hepatocytes and fibroblasts. We investigated the in vitro liver-specific function of cocultured hepatocytes long term for 1 month after primary hepatocyte isolation. The main goal of the study was to investigate whether multilayered hepatocyte sheet isogenic transplantation improves the liver-specific function and survival of rats with RILD in vivo.

\section{MATERIALS AND METHODS}

\section{Animals}

We used male F344 wild-type rats (male, 7-8 weeks, weighing 160-200 g) purchased from Japan SLC (Hamamatsu, Japan), both as donors (hepatocytes and fibroblasts) and recipients. The rats were housed in a temperaturecontrolled environment with a 12-h light/dark cycle and given free access to standard rat chow and water. In all procedures, the rats were anesthetized with either $2-2.5 \%$ isoflurane (Wako Pure Chemical Industries, Ltd., Osaka, Japan) inhalation or the intraperitoneal injection of pentobarbital sodium (1 ml/kg; Kyoritsu Seiyaku Corp. Tokyo, Japan). All experimental protocols were approved by the Animal Experimentation Committee of Nagasaki University.

\section{Hepatocyte Isolation}

Hepatocyte isolation was performed using a modified two-step collagenase perfusion method from donor rats according to previous reports (24). This procedure involved sequential perfusion of the liver with calcium-free Hank's balanced salt solution (HBSS; SigmaAldrich, St. Louis, MO, USA) and HBSS containing calcium (Sigma-Aldrich) and collagenase (130 U/ml; Wako Pure Chemical). The isolated cells were filtered through a cotton mesh membrane and 45- $\mu$ m stainless mesh and then purified three times via centrifugation at $50 \times g$ for 2 min each. Subsequently, the cell suspension was applied over $45 \%$ Percoll isodensity purification (GE Healthcare) via centrifugation at $50 \times g$ for $20 \mathrm{~min}$. In all experiments, we used only those hepatocytes exhibiting viability of at least $90 \%$, as estimated according to a trypan blue (Gibco, Life Technologies Corp., Carlsbad, CA, USA) exclusion test.

\section{Fibroblast Isolation}

Rat dermal fibroblasts (DFs) were isolated using a modified protocol according to a previous report (26). In brief, under sterile conditions, skin samples of approximately $1 \mathrm{~cm}^{2}$ were obtained from the underarm area of the donor rats. The tissue was divided into small pieces and then digested in $800 \mathrm{U} / \mathrm{ml}$ of collagenase type I (Worthington Biochemical Corp., Lakewood, NJ, USA) and $0.12 \mathrm{mg} / \mathrm{ml}$ of dispase (Gibco, Life Technologies Corp.) in a $37^{\circ} \mathrm{C}$ water bath for $40 \mathrm{~min}$. The digestion was stopped by adding $10 \mathrm{ml}$ of Minimum Essential Medium (MEM; Gibco, Life Technologies Corp.) supplemented with 10\% FBS (Gibco, Life Technologies Corp.), $2 \mathrm{mM}$ L-glutamine, $100 \mathrm{U} / \mathrm{ml}$ of penicillin, and $100 \mu \mathrm{g} / \mathrm{ml}$ of streptomycin (penicillin-streptomycinglutamine; Gibco, Life Technologies Corp.), and the supernatant was removed after centrifugation for two times at $500 \times g$ for $5 \mathrm{~min}$. The pellets were resuspended in $10 \mathrm{ml}$ of MEM and filtered through a $100-\mu \mathrm{m}$ nylon cell strainer (BD Falcon, Franklin Lakes, NJ, USA). The obtained supernatant was resuspended with MEM in a $10-\mathrm{cm}$ culture dish and then placed in a tissue culture incubator. For the experimental culture, DFs were used after at least five passages.

\section{Fabrication of Multilayered Hepatocyte Sheets}

Fabrication of the multilayered hepatocyte sheets was performed according to our previously described protocol $(22,23)$. In brief, on day 3 , the DF cell suspension was inoculated onto 35-mm temperature-responsive culture dish (TRCD) (UpCell; CellSeed Inc., Tokyo, Japan) at a density $2.0 \times 10^{5}$ cells/dish and cultured in MEM (Fig. 1). After the DFs formed a confluent monolayer within 3 days of culture, on day 0 , primary hepatocytes were isolated from the donor rats and inoculated onto the DF monolayer at a density of $8.0 \times 10^{5}$ cells/dish, after which they were cultured in Hepato-STIM medium (Hepatocyte Defined Medium; BD Biosciences, Franklin Lakes, NJ, USA) supplemented with $10 \% \mathrm{FBS}, 10 \mathrm{ng} / \mathrm{ml}$ of epidermal growth factor (EGF; Corning; Discovery Labware, Inc., Bedford, MA, USA), 2 mM L-glutamine, $100 \mathrm{U} / \mathrm{ml}$ of penicillin, and $100 \mu \mathrm{g} / \mathrm{ml}$ of streptomycin. On culture day 3 , the cocultured multilayered DFs and hepatocytes were detached as a cell sheet from the TRCD by lowering the culture temperature from $37^{\circ} \mathrm{C}$ to $20^{\circ} \mathrm{C}$ for $20 \mathrm{~min}$.

\section{Evaluation of the Liver-Specific Function In Vitro}

The rat albumin synthesis in the multilayered hepatocyte sheets was evaluated and compared with that observed in monolayered hepatocyte-only sheets as a control group 1 month after primary hepatocyte isolation. In order to make the monolayered hepatocyte-only sheets, on day 0 , primary hepatocytes were inoculated onto the FBS-coated TRCD at a density $8.0 \times 10^{5}$ cells/ dish. In both groups, the culture medium $(2 \mathrm{ml})$ was collected and replaced with new medium every $48 \mathrm{~h}$. Thereafter, the concentrations of the rat albumin in the 


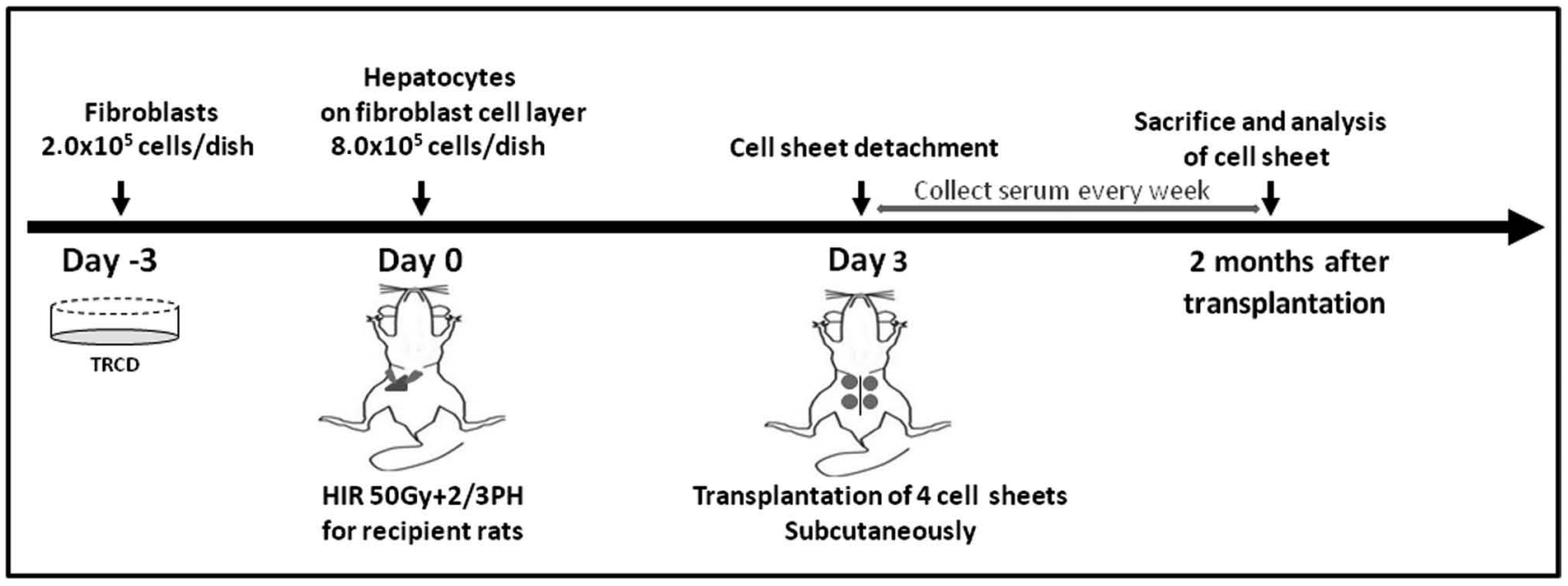

Figure 1. Protocol for multilayered hepatic cell sheet fabrication and transplantation in rats with RILD. Schematic representation of our experimental protocol.

culture medium were determined using an enzymelinked immunosorbent assay (ELISA). Goat anti-rat albumin $(6 \mu \mathrm{g} / \mathrm{ml})$ and peroxidase-conjugated sheep anti-rat albumin $(10 \mu \mathrm{g} / \mathrm{ml})$ antibodies (MP Biomedicals, LLC-Cappel products, Irvine, CA, USA) were used to detect rat albumin.

\section{RILD With PH and Multilayered Hepatocyte Sheets Transplantation}

The rats were randomly divided into two experimental groups: the transplant (RILD $+\mathrm{PH}+$ Transplantation) and control (RILD $+\mathrm{PH})$ groups. Each group consisted of 14 rats. In addition, a sham group was created in which the animals underwent laparotomy only $(n=5)$.

On day 0 , RILD with subsequent PH was performed in the rats (Fig. 1). In brief, after anesthesia was induced, the abdomen was opened via a midline incision and lead shields ( $2 \mathrm{~mm}$ thick) were gently placed to protect all other abdominal organs. Thereafter, RILD of the whole liver was performed using an X-ray irradiation device (ISOVOLT Titan 320; GE Inspection Technologies, Tokyo, Japan) at $50 \mathrm{~Gy}(320 \mathrm{kVP}, 5 \mathrm{~mA}$, and $0.5 \mathrm{~mm}$ $\mathrm{Cu}+0.5 \mathrm{~mm} \mathrm{Al}$ filtration at a dose rate of $0.87 \mathrm{~Gy} / \mathrm{min}$ ). Immediately after RILD, a standard PH of two thirds (median and left lateral liver lobes) was performed, and the abdomen was closed. In the transplant group, on day 3 (Fig. 1), four generated multilayered hepatocyte sheets were immediately transplanted into one recipient rat subcutaneously using a Cell Shifter (CellSeed Inc.) on both sides of the previously created midline incision. All four sheets were implanted separately from each other in the subcutaneous cavity. In the control group, RILD with PH was performed without further transplantation. The surviving rats in all groups were sacrificed at 2 months after transplantation.
For the assessment of the rat serum albumin concentrations, the sera from all rats were collected every week at the same time (from 9:00 to 10:00 am) for 2 months after transplantation, and the assessment was performed with ELISA using the same antibodies as was described earlier in the in vitro study.

\section{Morphological and Functional Investigations In Vivo}

Parts of the subcutaneous tissue areas treated with the transplanted multilayered hepatocyte sheets and whole livers were harvested at 2 months after transplantation. All tissue samples were fixed with $4 \%$ paraformaldehyde (Wako Pure Chemical Industries, Ltd.) in phosphate buffer solution and embedded in paraffin (Paraplast Plus; Leica Biosystems Richmond Inc., Richmond, IL, USA) with subsequent slicing into 5 - $\mu$ m-thick sections. Hematoxylin and eosin (H\&E; Muto Pure Chemicals Co., Ltd., Tokyo, Japan) staining was performed according to the standard staining protocol, and detection of the glycogen storage was performed using periodic acid-Schiff staining (PAS) according to the manufacturer's protocol (PAS staining kit; Merck KGaa, Darmstadt, Germany). For immunological staining, deparaffinized tissue sections were treated with either autoclave or microwave antigen retrieval in $1 \mathrm{mM}$ EDTA buffer solution in $10 \mathrm{mM}$ Tris-HCl (pH 9.0; Wako Pure Chemical Industries, Ltd.). Then the slides were allowed to cool at room temperature for approximately $1 \mathrm{~h}$ and blocked with $5 \%$ bovine serum albumin for $30 \mathrm{~min}$. The primary antibodies were anti-rat albumin (1:100 dilution), Ki-67 (1:100 dilution), CK8 (1:100 dilution), CK18 (1:100 dilution; all from Abcam, Cambridge, MA, USA), CD26 (1:100 dilution; Santa Cruz Biotechnology Inc., Dallas, TX, USA), and CD31 (1:50 dilution; Hycult Biotech Inc., Uden, Netherlands) incubated at $4^{\circ} \mathrm{C}$ overnight. Secondary antibodies (1:300 
dilution; all from Sigma Aldrich,) were incubated for $1 \mathrm{~h}$ at room temperature. The slides were then visualized with the 3,3'-diaminobenzidine (DAB) substrate-chromogen system (Dako, Carpinteria, CA, USA). The results of staining were assessed, and images were acquired using an inverted microscope (Nikon Eclipse Ti-U, Tokyo, Japan).

\section{Statistical Analysis}

All data are expressed as the mean \pm standard deviation and presented for at least six time points in two independent cell preparations. Comparisons between three groups were performed using the multiple comparisons test after a repeated measures two-way ANOVA analysis. A Kaplan-Meier log-rank analysis was performed for the survival study. The statistical analysis was performed using the GraphPad Prism 6 version software program (GraphPad Software, San Diego, CA, USA). In all analyses, $p<0.01$ and $p<0.05$ were taken to indicate statistical significance.

\section{RESULTS}

\section{Multilayered Hepatocyte Sheet Morphology}

Changes in the hepatocyte morphological characteristics on TRCD in each group are shown in Figure 2. On day 1, primary hepatocytes were found to be attached and spread on the FBS-coated TRCD as a flattened monolayer and demonstrated a cuboidal cell morphology with a bright intercellular border (Fig. 2A, B). On day 3, the hepatocytes formed a confluent monolayer; however, morphological degradation was evident compared with that seen in the day 1 culture (Fig. 2C). In the other group, on day 1 , after the primary hepatocytes adhered to the confluent DF cell monolayer, a spindle-like morphology repeating the shape of the DF cells in the more three-dimensional morphology was observed (Fig. 2E, F). On day 3, the multilayered hepatocyte sheets reached confluence with a maintained morphology, while most of the hepatocytes remained viable and attached (Fig. 2G).

Furthermore, on day 3 , the primary hepatocytes under both conditions were detached as cell sheets from the TRCD. It should be noted that for monolayered hepatocyte sheet formation, $3-5 \mathrm{~h}$ of incubation at $20^{\circ} \mathrm{C}$ was needed, and further gentle pipetting was required due to the high fragility to achieve complete detachment (Fig. 2D). In contrast, for multilayered hepatocyte sheet formation, 20 min was enough, without the need for further pipetting for complete detachment, and the structure of the cell sheet was stronger due to the greater thickness (Fig. 2H).

\section{Albumin Concentrations in the Culture Medium}

Changes in the albumin synthesis rate in vitro in each group are shown in Figure 3. On culture day 3, the albumin synthesis activity was not significantly different between the two groups; however, the levels in the multilayered

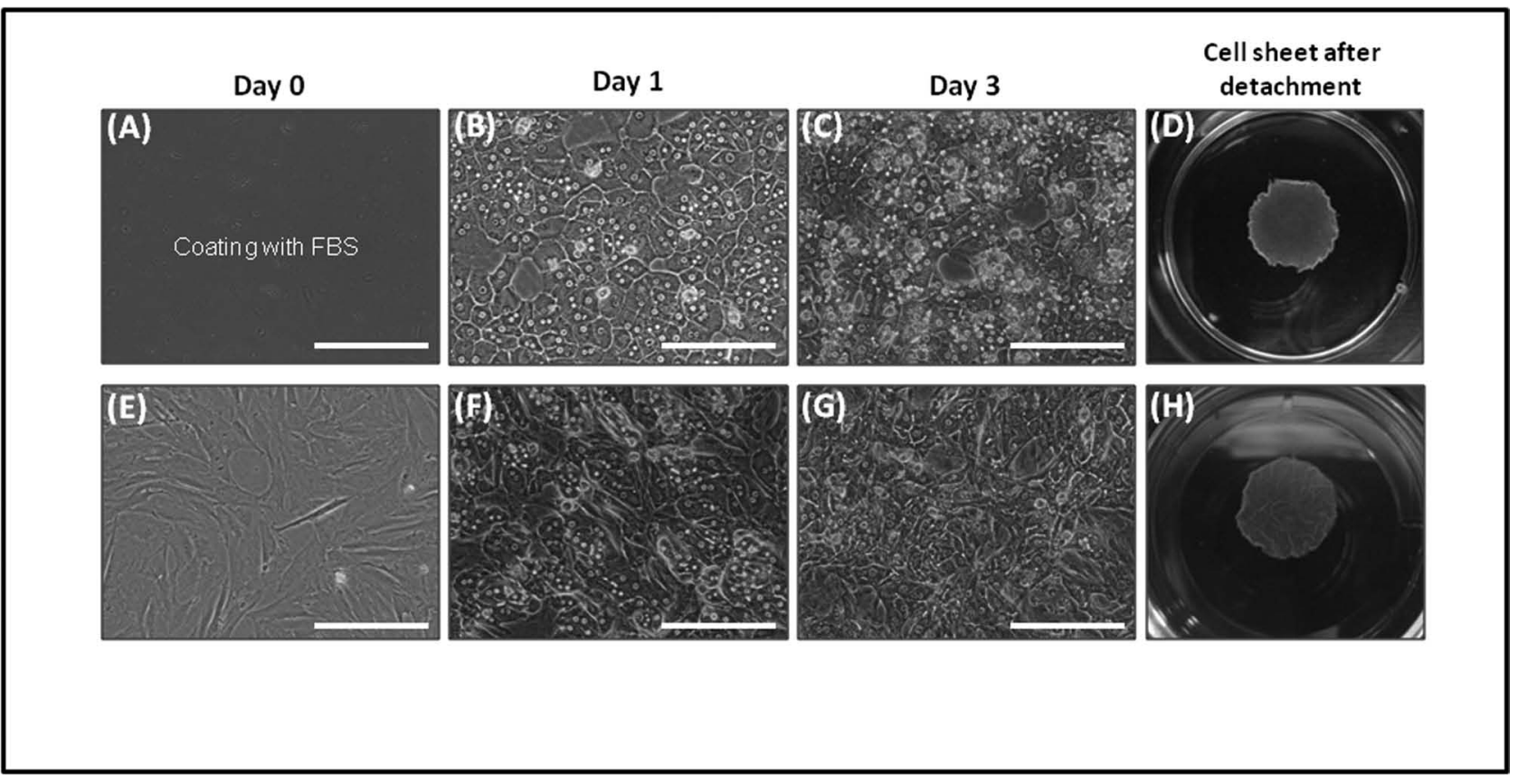

Figure 2. Cell morphologies of hepatocytes treated with and without fibroblast coculture on TRCD. Phase-contrast micrographs show the morphology of the monolayered $(A-C)$ and multilayered $(E-G)$ hepatocytes on days: 0 (A, E), 1 (B, F), and 3 (C, G). Macroscopic observation of the fabricated monolayered $(\mathrm{D})$ and multilayered $(\mathrm{H})$ hepatocyte sheets after detachment from the TRCD. Scale bars: $200 \mu \mathrm{m}$. 


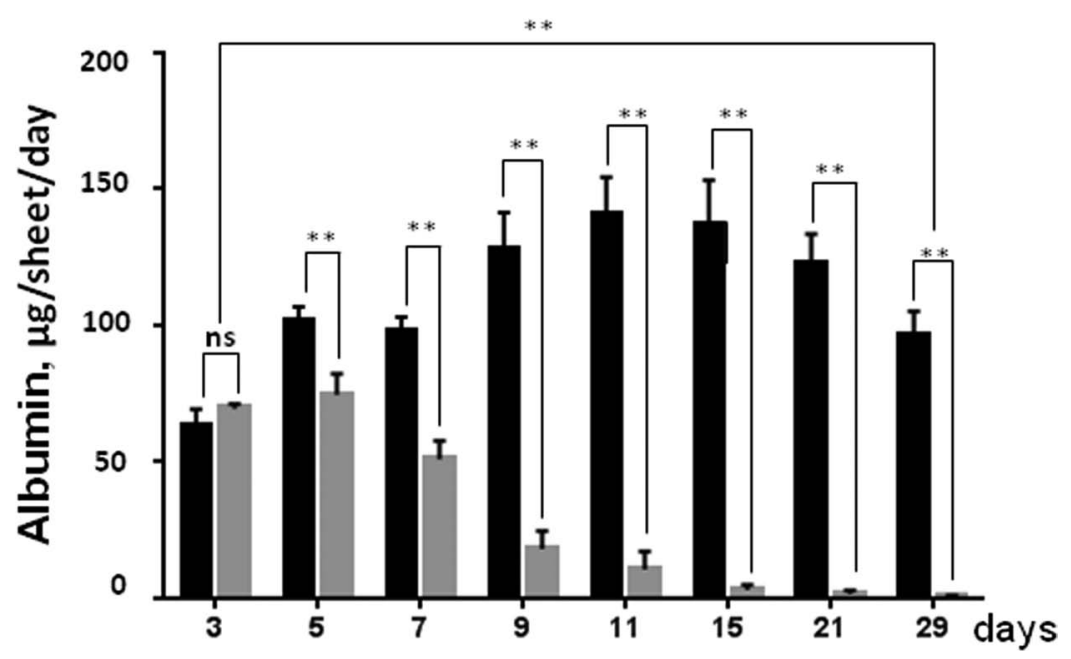

Figure 3. In vitro comparison of the rate of albumin synthesis between the multilayered and monolayered hepatocytes. Quantitation using ELISA revealed significantly high albumin concentrations in the culture medium of the multilayered hepatocytes (black bars) in comparison with the monolayered hepatocytes (gray bars) during the observation period, $* * p<0.01$.

hepatocyte sheet group (black bars) started to continuously increase and reached the highest peak on day 11 , at which time they were significantly higher than that seen under the control conditions $(140.33 \pm 13.9$ vs. $10.5 \pm 6.5$ $\mu \mathrm{g} / \mathrm{sheet} / \mathrm{day} ; p<0.01)$. Subsequently, the albumin levels in the multilayered hepatocyte sheets began to slowly decrease and showed a value of $95.9 \pm 8.9 \mu \mathrm{g} / \mathrm{sheet} /$ day after 1 month of observation, whereas the levels in the monolayered hepatocyte sheets were only $0.5 \pm 0.3 \mu \mathrm{g} /$ sheet/day $(p<0.01)$. Nevertheless, even after 1 month of culture, the levels of albumin synthesis in the multilayered hepatocyte sheets in vitro were significantly higher than those observed initially on day $3(95.9 \pm 8.9$ vs. $62.9 \pm 6.4 \mu \mathrm{g} / \mathrm{sheet} / \mathrm{day} ; p<0.01$ ).

The multilayered hepatocyte sheets had a significantly greater hepatocyte-specific function and maintained this function in vitro for at least 1 month after primary isolation. In addition, after 30 days in culture, the morphology of the primary isolated multilayered hepatocytes was maintained, and most of the hepatocytes remained viable and attached (Fig. 4A). Moreover, after 30 days of culture, it was still easy to fabricate cell sheets, and the storage of the glycogen and albumin in the cell sheets was confirmed immunohistochemically (Fig. 4B-D).

\section{Morphological Characteristics of the Multilayered Hepatocyte Sheets After Transplantation}

At 2 months after transplantation, the multilayered sheets proliferated, and multiple new tissue layers with hepatocytes were observed in the subcutaneous cavity in the recipient rats, which expressed the hepatocyte-specific marker CK8/18, as confirmed by immunohistochemistry (Fig. 5A, B). Furthermore, the transplanted multilayered sheets showed the storage of glycogen (Fig. 5C) and albu$\min$ (Fig. 5D) and the existence of bile canaliculus structures (Fig. 5E). Some hepatocytes and fibroblasts were still positive for the proliferation marker Ki-67 (Fig. 5F). Moreover, multiple areas of vascularization around and permeated inside the cell sheets were observed (Fig. 5G, H).

These data show the possibility that the transplanted multilayered sheets respond to regenerative stimuli after performing RILD with PH. Moreover, the further proliferative activity of the hepatocytes with maintenance of their functional ability and a healthy morphology were confirmed.

\section{Serum Albumin Concentrations}

After performing RILD and $\mathrm{PH}$, in both groups, the serum albumin concentrations declined sharply (Fig. 6A); however, in the transplant group, the levels of serum albumin reached the lowest level at 1 week and then started to recover, being significantly higher than that seen in the control group by 5 weeks after transplantation $(41.0 \pm 11.84$ vs. $26.4 \pm 7.14 \mathrm{mg} / \mathrm{ml} ; p<0.01)$. After an observation period of 2 months, the serum albumin levels remained significantly higher than those in the control group $(54.3 \pm 9.6$ vs. $32.7 \pm 5.7 \mathrm{mg} / \mathrm{ml} ; p<0.01)$ and were comparable to the serum albumin levels in the normal rats $(58.2 \pm 6.4 \mathrm{mg} / \mathrm{ml})$ (Fig. 6A). The serum albumin levels were significantly different between the control and sham groups during all observation periods, except on day -3 (Fig. 6A).

\section{Survival Rate}

After the 63-day observation period, only $22 \%$ of the rats survived in the control group, in which the rats received only RILD and PH (Fig. 6B). In the transplant group, treatment with the transplanted multilayered hepatocyte 

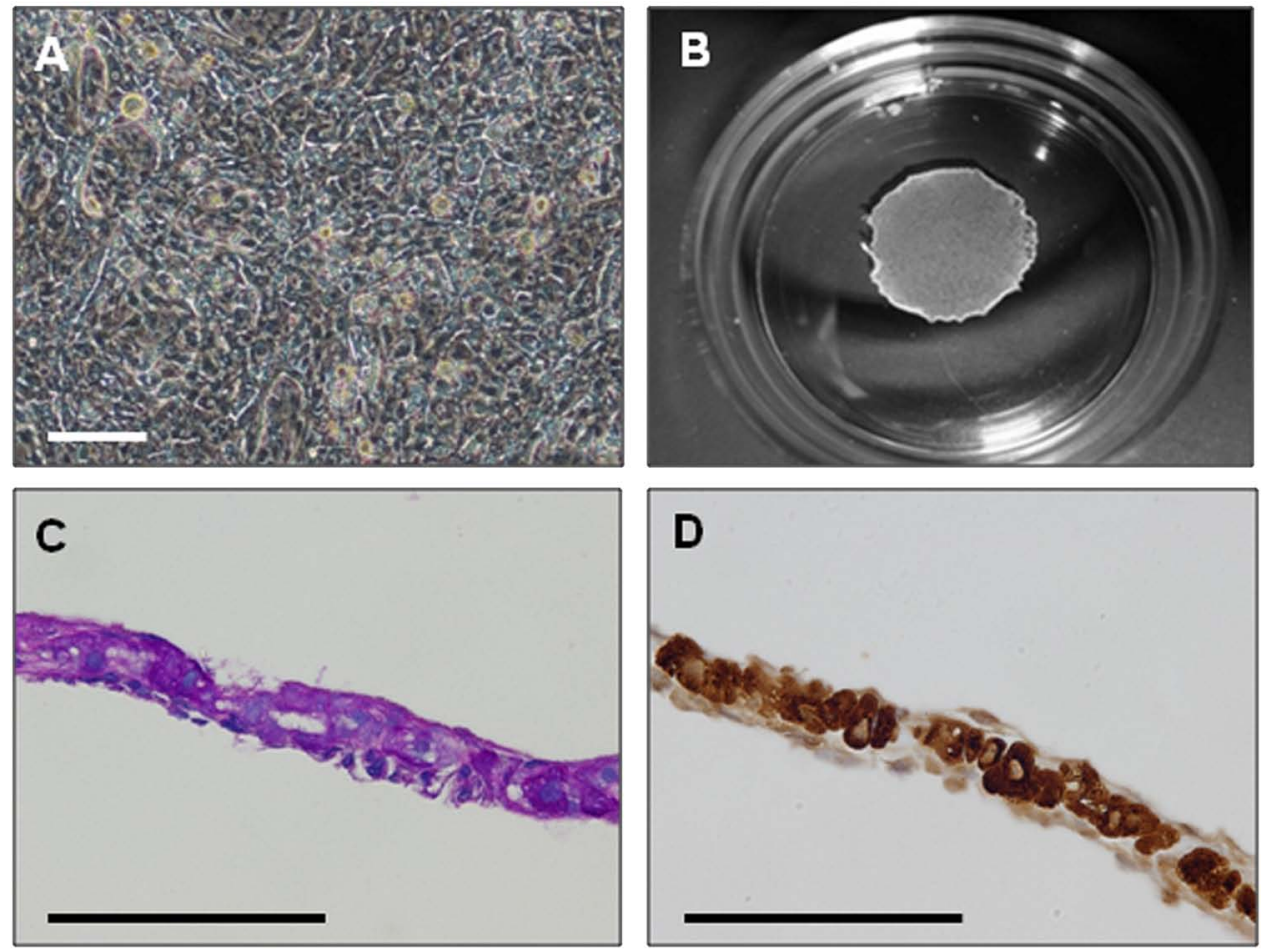

Figure 4. Morphological changes in the multilayered hepatocytes and fabricated cell sheets after 30 days in culture in vitro. (A) The morphology of the multilayered hepatocytes was maintained, and most of the hepatocytes remained viable and attached. The fabricated multilayered hepatocyte sheets after 30 days (B) showed glycogen storage (C) and albumin synthesis (D). Scale bars: $100 \mu \mathrm{m}$.

sheets significantly improved the survival rate of the rats receiving RILD and $2 / 3 \mathrm{PH}$ up to $57 \%(p<0.05)$.

\section{Analysis of the Liver Function}

Figure 7 shows the morphological and functional changes in the liver after the 2-month observation period. In both the transplant and control groups, H\&E staining showed severe histopathological changes, with liver cell atrophy and a reduction in the number of hepatocytes (Fig. 7A, D). Massive portal and sinusoidal fibrosis with an expanded portal tract and bile duct proliferation were also observed. In addition, in both groups, large numbers of hepatocytes were negative for glycogen and albumin (Fig. 7B, C and E, F). In contrast, the examination of the liver tissues from all sham rats revealed a normal cellular architecture and functionality with a widespread glycogen and albumin expression (Fig. 7G-I).

\section{DISCUSSION}

The first animal experiments showed that suspension of hepatocytes subcutaneously transplanted into syngeneic rats can maintain specific histochemical differentiation with the formation of bile canalicular structures in extrahepatic sites for prolonged periods (14), and the stimulus with partial hepatectomy enhanced the probability of nodule formation in hepatocytes (15). On the other hand, we previously reported (23) that multilayered hepatocyte sheets show significantly higher liver specific function in vivo compared to the cosuspension of hepatocytes and fibroblasts. Thus, liver tissue engineering has become an attractive target for regenerative medicine. The properties of cell sheets obtained via engineering using hepatocytes have recently been characterized in both in vitro and in vivo studies as well $(16,20-23)$. However, no studies have successfully investigated the treatment of liver disease in models utilizing cell sheet engineering therapy. The current study showed that multilayered hepatocyte sheets generated from isogenic primary hepatocytes and primary skin fibroblasts are able to proliferate after transplantation into the subcutaneous cavity in a diseased rat model and thereby support the host liver function while improving the serum albumin level up to the normal range within 2 months. The improvement in the survival rate, which was the second main goal of the current study, indeed indicates the high potential of hepatocyte cell sheet therapy.

Previous studies have demonstrated various methods of coculturing hepatocytes with fibroblasts that enhance the hepatocyte function and viability compared to cultures of hepatocytes only $(3,4,18)$. Although the detailed mechanisms of the cell-cell interactions between hepatocytes and fibroblasts have not been investigated, it is assumed that fibroblasts express essential cytokines and extracellular 

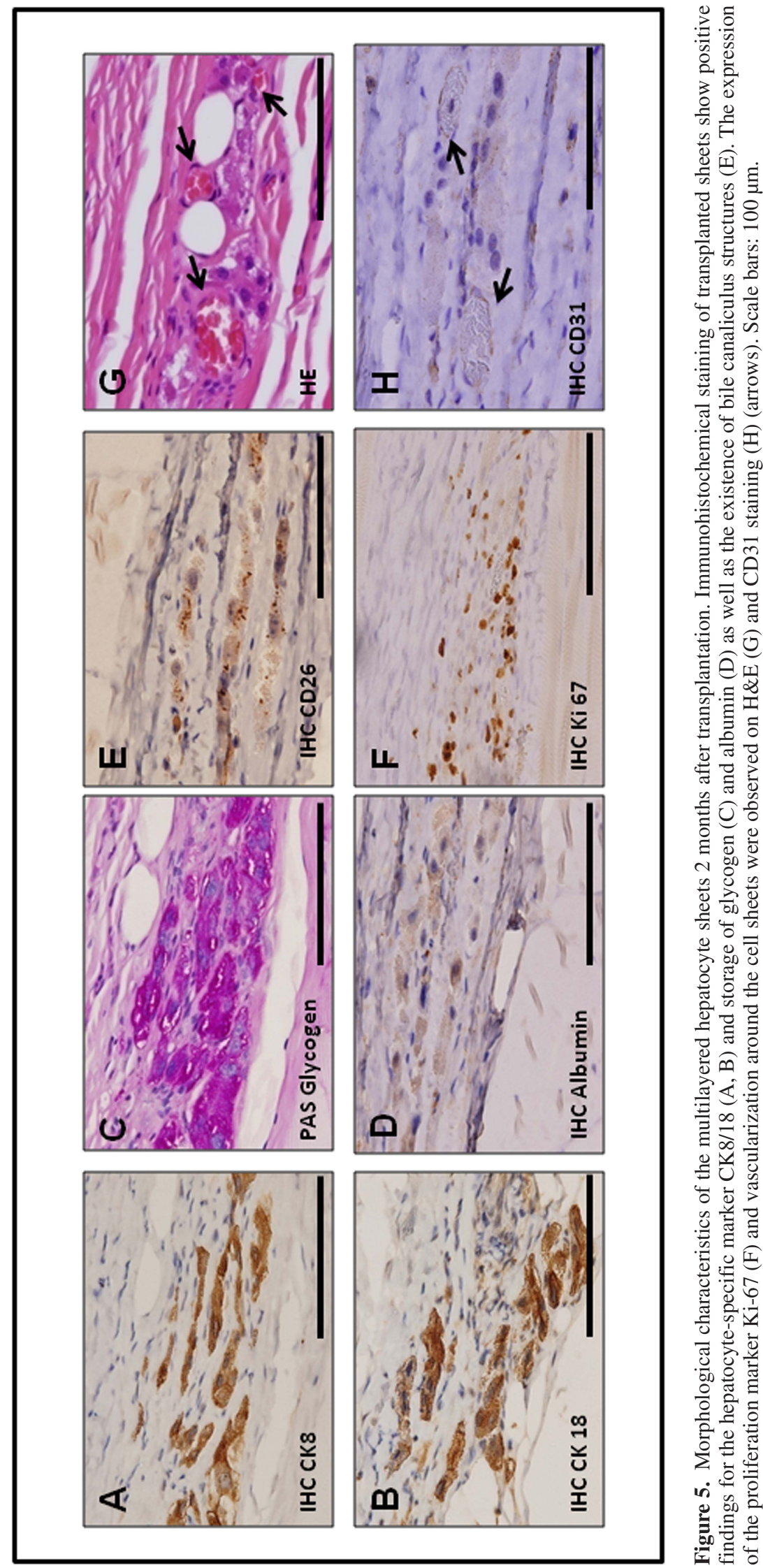
A

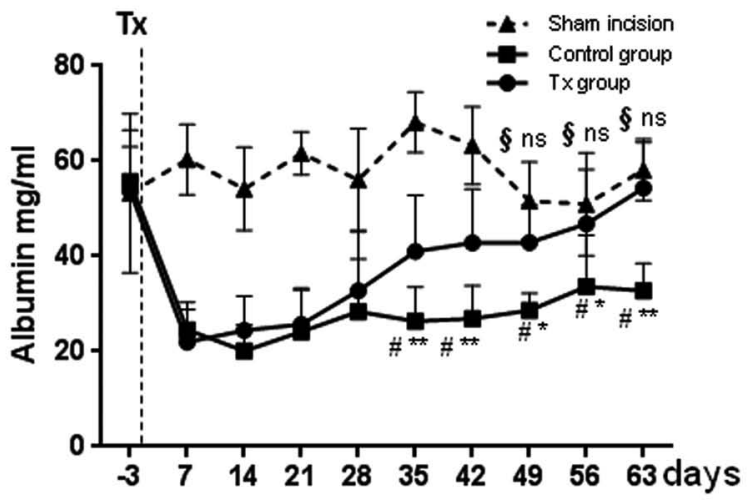

B

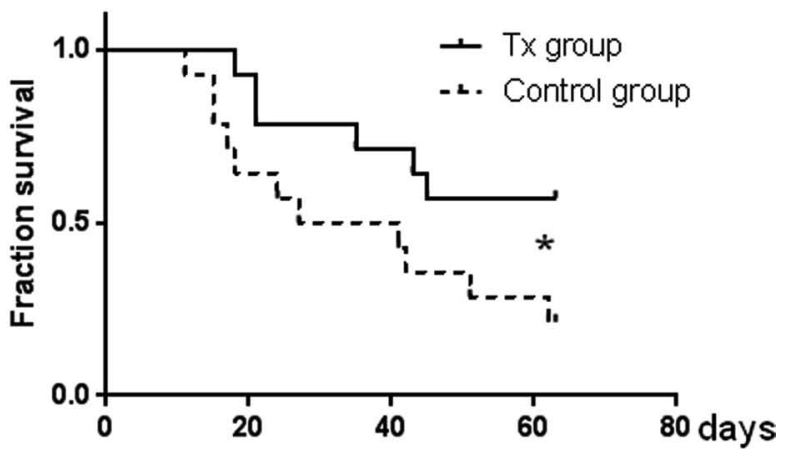

Figure 6. Analysis of the liver function and survival rate after transplantation. (A) Comparison of the serum albumin concentrations between the groups assessed using ELISA during the 2-month observation period (\#Transplant vs. Control, §Transplant vs. Sham; $* p<0.05, * * p<0.01)$. The levels of serum albumin were significantly different between the Sham and Control groups during all observation periods, except on day -3. (B) The Kaplan-Meier survival curve shows that treatment with the transplanted hepatocyte sheets significantly improved the survival rate of the rats receiving RILD and 2/3PH (log-rank test $* p<0.05$ ).

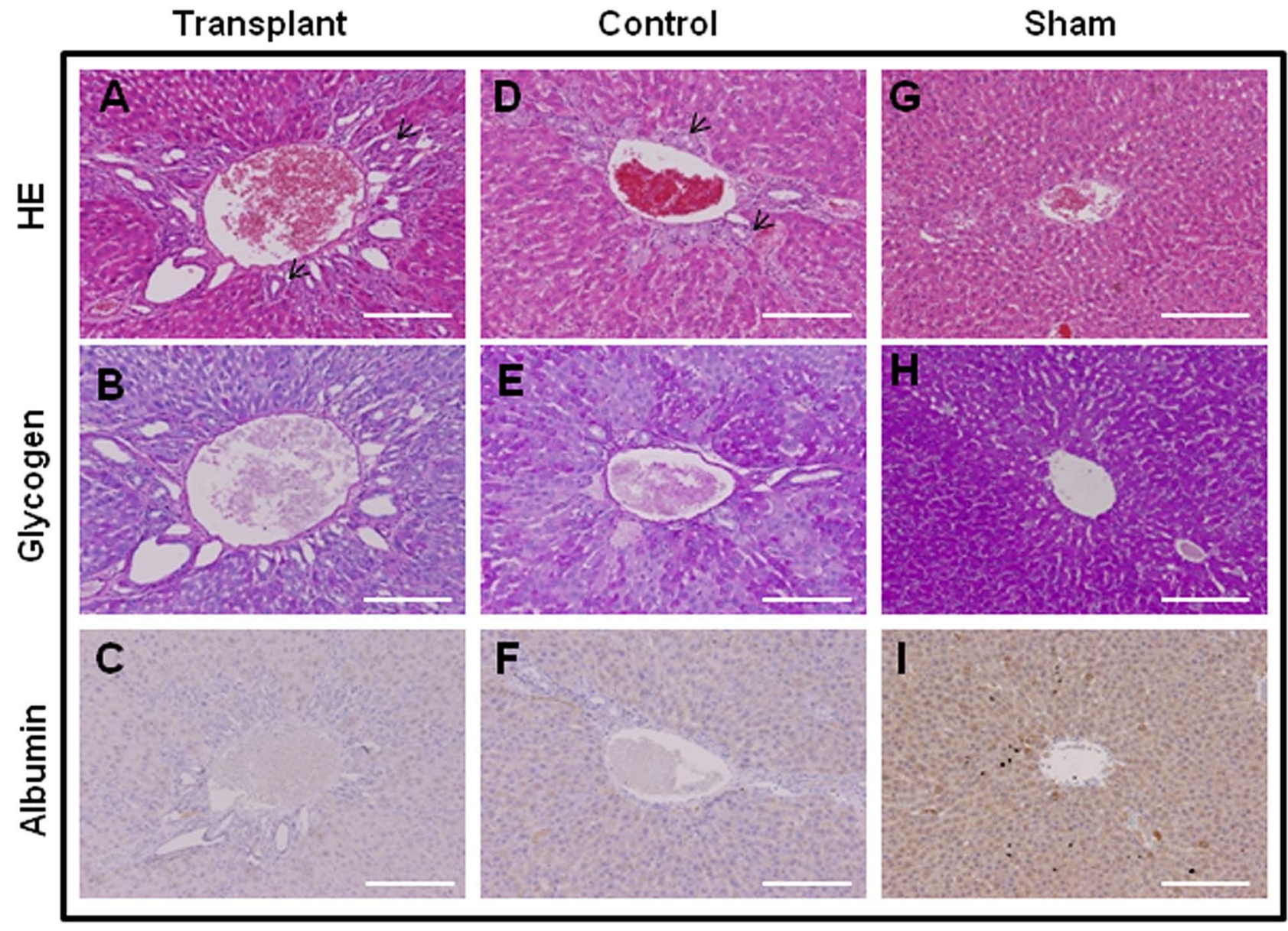

Figure 7. Morphological and functional characteristics of the liver 2 months after transplantation. H\&E shows severe histopathological changes in the transplant (A) and control (D) groups. Massive portal and sinusoidal fibrosis with an expanded portal tract and bile duct proliferation (black arrowheads) were observed. In both groups, large numbers of hepatocytes were negative for glycogen (B, E) and albumin (C, F). In contrast, the sham group showed a normal cellular architecture and functional capacity (G, H, I). Scale bars: $200 \mu \mathrm{m}$. 
matrix components following direct contact with hepatocytes $(2,11,23)$. These heterotypic cellular interactions have been shown to play essential roles in improving the hepatocyte function. As we described previously, our technique of fabricating multilayered hepatic cell sheets using fibroblasts as feeder cells has the additional advantage of preserving high viability and functionality in vitro (22) and in vivo (23). Considering the large place for transplantation in the subcutaneous cavity and the ability to fabricate adequate amounts of cell sheets, it is not necessary to achieve layer-by-layer sheet deposition, high-density cultures, or cell proliferation. Moreover, after cell sheet detachment from the TRCD, no destruction of the originating cell-cell and cell-extracellular matrix interactions formed during cocultivation was observed, which is essential for high functionality and structural support.

We performed RILD in the recipient animals in order to inhibit the proliferative and regenerative capacity of the host hepatocytes after PH and, at the same time, provide transplanted cells for further proliferation. At 2 months after transplantation, newly proliferated multiple hepatocyte layers were observed subcutaneously, although initially the cell sheet consisted of only one layer of hepatocytes. Furthermore, after 2 months, large numbers of Ki-67positive hepatocytes remained. A similar phenomenon has been previously reported, wherein long-term proliferation (40 weeks) of host hepatocytes is evident and confirmed based on an elevated Ki-67 labeling index following lower dose (30 Gy) RILD in a rat model (12). Therefore, we speculate that after severe damage to host hepatocytes induced by 50 Gy of RILD, the remaining proliferative impact in the host liver influences the transplanted hepatocytes to proliferate for a long time after transplantation.

Hypoxia is a major cause of poor hepatocyte survival soon after transplantation. In the current study, we observed vascularization in and around the cell sheets in the subcutaneous cavity only 1 week after transplantation (data not shown). Therefore, immediately after transplantation, all transplanted cells are supplied only with oxygen from surface diffusion because of the lack of capillary vessels, and the tissue size of the cell aggregates and thickness of the cell tissues is very important in terms of cell viability and functionality $(22,23,25,27)$. After 2 months, the transplanted hepatocyte sheets were permeated with multiple capillary vessels. Therefore, the hepatocytes were close to blood vessels, confirming that vascularization is crucial for their survival and function.

We confirmed that the multilayered hepatic cell sheets showed dramatically higher albumin expression levels in vitro during 1 month after transplantation compared to that seen in the cultures of hepatocytes only, and after the observation period it was still easy to fabricate and handle the cell sheets. These findings provide a large opportunity to prolong the use of primary isolated hepatocytes while preserving high viability and metabolic functionality, which remain as unsolved problems for HT, requiring the immediate transplantation of primary hepatocytes. In recent years, HT-based procedures have been established and increasingly characterized $(1,6-8,13,17,19,25)$. However, there continue to be many limitations preventing their wide use $(9,10,28)$. Only a small amount of hepatocytes can be transplanted into the portal system at one time in order to prevent serious complications, such as portal vein thrombosis, portal hypertension resulting from obstruction of the sinusoids and hepatic infarction $(9,10)$. Furthermore, most of the transplanted hepatocytes die due to anoikis, hypoxia, or phagocytosis. In contrast, our technique allows for easy fabrication and implantation of a sufficient amount of hepatocyte sheets, as we achieved with the transplantation of four cell sheets $\left(4.0 \times 10^{6}\right.$ cells $)$ into each recipient rat. In addition, after HT, there is no possibility of controlling the transplanted hepatocytes with further histological analyses, which may be necessary in cases of cellular rejection after transplantation (13). Despite the fact that the liver is considered an optimal place for transplantation, the implantation of cell sheets into the subcutaneous cavity has advantages in terms of minimizing invasiveness, monitoring the transplanted cells, and promptly removing the cell sheets in cases of rejection. Therefore, the subcutaneous cavity may be considered the optimal place for transplantation in the era of cell sheet-based therapy.

We believe that hepatocyte sheet transplantation technology offers a great therapeutic opportunity both for the treatment of metabolic liver diseases and providing support for the liver function in patients with end-stage liver failure. It has been reported that the replacement of approximately $5 \%$ of a theoretical liver mass may restore a sufficient metabolic function in humans $(5,10)$. However, in addition to the above-mentioned problems in the field of HT, it still remains unclear as to precisely how many functioning engrafted cells remain after HT (10). Therefore, further studies in large-animal models should be conducted to determine the sufficient number and size of required cell sheets to perform successful transplantation in humans and also confirm the effectiveness of cell sheet technology in future clinical applications. In addition, several liver failure models should be performed including a mild liver disease status, to clarify the potential of the liver disease treatment.

In conclusion, the current observations suggest that transplanted multilayered hepatocyte sheets maintain the liver-specific function with a further proliferative activity in vivo for at least 2 months and thus provide metabolic support in rats with radiation-induced liver damage. We speculate that the further development of this protocol will have great potential in the field of cell-based therapy for metabolic liver disease. 
ACKNOWLEDGMENTS: This study was supported in part by Grants-in-Aid for Young Scientists to Y. Sakai (No. 25861161) and Scientific Research to S. Eguchi (No.23591868 and No. 26461916), and Takeda Science Foundation to Y. Sakai. The authors declare no conflicts of interest.

\section{REFERENCES}

1. Arkadopoulos, N.; Chen, S. C.; Khalili, T. M.; Detry, O.; Jewitt, W. R.; Lila, H.; Kamachi, H.; Petrovic, L.; Mullon, C. J.; Demetriou, A. A.; Rozga, J. Transplantation of hepatocytes for prevention of intracranial hypertension in pigs with ischemic liver failure. Cell Transplant. 7:357-363; 1998.

2. Bhatia, S. N.; Balis, U. J.; Yarmush, M. L.; Toner, M. Effect of cell-cell interactions in preservation of cellular phenotype: Cocultivation of hepatocytes and nonparenchymal cells. FASEB J. 13:1883-1900; 1999.

3. Bhatia, S. N.; Balis, U. J.; Yarmush, M. L.; Toner, M. Microfabrication of hepatocyte/fibroblast co-cultures: Role of homotypic cell interactions. Biotechnol. Prog. 14:378387; 1998.

4. Cho, C. H.; Park, J.; Tilles, A. W.; Berthiaume, F.; Toner, M.; Yarmush, M. L. Layered patterning of hepatocytes in coculture systems using microfabricated stencils. Biotechniques 48:47-52; 2010

5. Filippi, C.; Dhawan, A. Current status of hepatocyte transplantation and its potential for Wilson's disease. Ann. NY Acad. Sci. 1315:50-55; 2014.

6. Fox, I. J.; Chowdhury, J. R.; Kaufman, S. S.; Goertzen, T. C.; Chowdhury, N. R.; Warkentin, P. I.; Dorko, K.; Sauter, B. V.; Strom, S. C. Treatment of the Crigler-Najjar syndrome type I with hepatocyte transplantation. N. Engl. J. Med. 338:1422-1426; 1998.

7. Guha, C.; Sharma, A.; Gupta, S.; Alfieri, A.; Corla, G.; Gagandeep, S.; Sokhi, R.; Roy-Chowdhury, N.; Tanaka, K. E.; Vikram, B.; Roy-Chowdhury, J. Amelioration of radiation induced liver damage in partially hepatectomized rats by hepatocyte transplantation. Cancer Res. 59:5871-5874; 1999.

8. Gupta, S.; Rajvanshi, P.; Sokhi, R.; Slehria, S.; Yam, A.; Kerr, A.; Novikoff, P. Entry and integration of transplanted hepatocytes in rat liver plates occur by disruption of hepatic sinusoidal endothelium. Hepatology 29:509-519; 1999.

9. Holzman, M. D.; Rozga, J.; Neuzil, D. F.; Griffin, D.; Moscioni, A. D.; Demetriou, A. A. Selective intraportal hepatocyte transplantation in analbuminemic and Gunn rats. Transplantation 55:1213-1219; 1993.

10. Hughes, R. D.; Mitry, R. R.; Dhawan, A. Current status of hepatocyte transplantation. Transplantation 93:342-347; 2012.

11. Hui, E. E.; Bhatia, S. N. Micromechanical control of cellcell interactions. Proc. Natl. Acad. Sci. USA 104:57225726; 2007.

12. Imaeda, M.; Ishikawa, H.; Yoshida, Y.; Takahashi, T.; Ohkubo, Y.; Musha, A.; Komachi, M.; Nakazato, Y.; Nakano, T. Long-term pathological and immunohistochemical features in the liver after intraoperative whole-liver irradiation in rats. J. Radiat. Res. 55:665-673; 2014.

13. Ito, M.; Nagata, H.; Miyakawa, S.; Fox, I. Review of hepatocyte transplantation. J. Hepatobiliary Pancreat. Surg. 16:97-100; 2009

14. Jirtle, R. L.; Biles, C.; Michalopoulos, G. Morphologic and histochemical analysis of hepatocytes transplanted into syngeneic hosts. Am. J. Pathol. 101:115-126; 1980.
15. Jirtle, R. L.; Michalopoulos, G. Effects of partial hepatectomy on transplanted hepatocytes. Cancer Res. 42:3000$3004 ; 1982$

16. Kim, K.; Ohashi, K.; Utoh, R.; Kano, K.; Okano, T. Preserved liver-specific functions of hepatocytes in 3D coculture with endothelial cell sheets. Biomaterials 33:14061413; 2012.

17. Krause, P.; Wolff, H. A.; Rave-Frank, M.; Schmidberger, H.; Becker, H.; Hess, C. F.; Christiansen, H.; Koenig, S. Fractionated external beam radiotherapy as a suitable preparative regimen for hepatocyte transplantation after partial hepatectomy. Int. J. Radiat. Oncol. Biol. Phys. 80:12141219; 2011.

18. Lu, H. F.; Chua, K. N.; Zhang, P. C.; Lim, W. S.; Ramakrishna, S.; Leong, K. W.; Mao, H. Q. Threedimensional co-culture of rat hepatocyte spheroids and $\mathrm{NIH} / 3 \mathrm{~T} 3$ fibroblasts enhances hepatocyte functional maintenance. Acta Biomater. 1:399-410; 2005.

19. Minato, M.; Houssin, D.; Demma, I.; Morin, J.; Gigou, M.; Szekely, A. M.; Bismuth, H. Transplantation of hepatocytes for treatment of surgically induced acute hepatic failure in the rat. Eur. Surg. Res. 16:162-169; 1984.

20. Ohashi, K.; Tatsumi, K.; Tateno, C.; Kataoka, M.; Utoh, R.; Yoshizato, K.; Okano, T. Liver tissue engineering utilizing hepatocytes propagated in mouse livers in vivo. Cell Transplant. 21:429-436; 2012.

21. Ohashi, K.; Yokoyama, T.; Yamato, M.; Kuge, H.; Kanehiro, H.; Tsutsumi, M.; Amanuma, T.; Iwata, H.; Yang, J.; Okano, T.; Nakajima, Y. Engineering functional two- and threedimensional liver systems in vivo using hepatic tissue sheets. Nat. Med. 13:880-885; 2007.

22. Sakai, Y.; Koike, M.; Hasegawa, H.; Yamanouchi, K.; Soyama, A.; Takatsuki, M.; Kuroki, T.; Ohashi, K.; Okano, T.; Eguchi, S. Rapid fabricating technique for multi-layered human hepatic cell sheets by forceful contraction of the fibroblast monolayer. PLoS One 8:e70970; 2013.

23. Sakai, Y.; Yamanouchi, K.; Ohashi, K.; Koike, M.; Utoh, R.; Hasegawa, H.; Muraoka, I.; Suematsu, T.; Soyama, A.; Hidaka, M.; Takatsuki, M.; Kuroki, T.; Eguchi, S. Vascularized subcutaneous human liver tissue from engineered hepatocyte/fibroblast sheets in mice. Biomaterials 65:66-75; 2015 .

24. Seglen, P. O. Preparation of isolated rat liver cells. Methods Cell. Biol. 13:29-83; 1976.

25. Sekine, W.; Haraguchi, Y.; Shimizu, T.; Umezawa, A.; Okano, T. Thickness limitation and cell viability of multilayered cell sheets and overcoming the diffusion limit by a porous-membrane culture insert. J. Biochip Tissue Chip S1:007; 2011

26. Seluanov, A.; Vaidya, A.; Gorbunova, V. Establishing primary adult fibroblast cultures from rodents. J. Vis. Exp. 44:2033; 2010

27. Tamura, T.; Sakai, Y.; Nakazawa, K. Two-dimensional microarray of HepG2 spheroids using collagen/polyethyl polyethylene glycol micropatterned chip. J. Mater. Sci. Mater. Med. 19:2071-2077; 2008.

28. Terry, C.; Mitry, R.; Lehec, S.; Muiesan, P.; Rela, M.; Heaton, N.; Hughes, R. D.; Dhawan. A. The effects of cryopreservation on human hepatocytes obtained from different sources of liver tissue. Cell Transplant. 14:585$594 ; 2005$. 